\title{
Editorial: Advances and Challenges in Ocean Wave Energy Harvesting
}

\author{
Yingyi Liu ${ }^{1}$, Boyin Ding ${ }^{2}$, Binzhen Zhou ${ }^{3}$, Peiwen Cong ${ }^{4}$ and Siming Zheng ${ }^{5 *}$ \\ ${ }^{1}$ Research Institute for Applied Mechanics, Kyushu University, Fukuoka, Japan, ${ }^{2}$ Ocean Wave Energy Research Group, School of \\ Mechanical Engineering, Faculty of Engineering, Computer and Mathematical Sciences, University of Adelaide, Adelaide, SA, \\ Australia, ${ }^{3}$ School of Civil Engineering and Transportation, South China University of Technology, Guangzhou, China, ${ }^{4}$ State Key \\ Laboratory of Coastal and Offshore Engineering, Dalian University of Technology, Dalian, China, ${ }^{5}$ School of Engineering, \\ Computing and Mathematics, University of Plymouth, Plymouth, United Kingdom
}

Keywords: wave energy, wave farm, floating converter system, hybrid energy converters, control strategy, numerical modeling

\section{Editorial on the Research Topic}

\section{Advances and Challenges in Ocean Wave Energy Harvesting}

Wave energy resource is abundant and huge due to the vast oceanic area on the planet. On the other hand, exploitation of wave energy has never reached a satisfactory level that can replace to a large extent the traditional fossil fuel. The main disadvantage of wave power is the significantly random variability in several timescales: from wave to wave, with sea state, and from month to month (Falcão, 2010). This poses many super challenges to effectively and efficiently extract power from the sea waves.

The present collection of research articles reflects the most recent contributions coming forward on harvesting wave energy, six coming from China (which is not completely surprising because of China's massive investments in recent years) and one from Sweden. They include six technical articles (Ning et al., 2019; Zhang et al., 2019; Qiao et al., 2020; Tan et al., 2020; Wang et al., 2020; Zhou et al., 2020) and one comprehensive review (Malin et al., 2020). These research works cover several representative types of wave energy converters, including OWC (oscillating water columns) (Ning et al., 2019; Qiao et al., 2020), PA (point absorber) (Tan et al., 2020; Zhou et al., 2020), and Edinburgh Duck WEC (wave energy converter) (Zhang et al., 2019). Numerical investigation (Ning et al., 2019; Zhang et al., 2019; Malin et al., 2020; Qiao et al., 2020; Tan et al., 2020; Wang et al., 2020; Zhou et al., 2020), experimental study (Wang et al., 2020), and open sea test (Zhang et al., 2019) have all been performed in the trials on modeling either a single WEC or wave farms (Malin et al., 2020). In addition, the effort to the evaluation of the sea climate has also been made (Wang et al., 2020).

Zhang et al. (2019) developed a hybrid boundary element method applying eigenfunction expansion in the external domain and numerically compute the optimal capture width ratio of their modified Duck device based on the Edinburgh Duck WEC. Besides, Zhang et al. (2020) also show some measurement data of their open sea tests on the modified Duck device.

Ning et al. (2019) applied a fully nonlinear higher-order boundary element method and numerically investigate the influence of the step bottom configuration on the efficiency of an OWC. Experimental and CFD results in the literature have also been compared with, and good agreement is found. It is concluded that higher operational efficiency can be achieved by optimizing the step geometry and position for a given wave condition.

Tan et al. (2020) perform a parametric study on a two-body wave energy point absorber. They made substantial linearization on their nonlinear time-domain model in order to improve efficiency. Their case study suggests that utilizing sufficient small stiffness of the power take-off system and the floater and optimal mass of the bodies can help to achieve the maximum power output.

Malin et al. (2020) perform a very comprehensive review of the state of the art of wave energy park optimization, involving modeling methods, experiments, and optimization algorithms. Moreover, a set of 
impressive results have been presented to analyze how realistic, reliable, and relevant the methods and the results are. The readers are strongly recommended to read the elaborate contents of the study, which may enlighten good ideas on further exploring new directions.

Qiao et al. (2020) discuss the possibility of integrating an OWC with an offshore jacket platform. A numerical study based on the finite element method has been performed for the dynamic analysis of such a combination. It is found that the affiliated OWC device can bring green wave energy while causing an almost negligible effect on the dynamic responses.

Wang et al. (2020) carry out a physical experiment in the wave flume in investigating the generation process of focused waves using double wave groups with different peak frequency differences. It is found that the phase shifts are mainly caused by the third-order nonlinearity due to interactions between the two wave groups. More investigations are conducted with numerical simulations based on the high-order spectral (HOS) method on the evolution of freak waves in the real sea environment.

Zhou et al. (2020) develop a numerical method that supplements the potential flow theory with a viscous correction. They thereby apply this method to study the motion response and performance of a heaving point absorber WEC with flat, cone, and hemispherical bottoms. After calibration with CFD results, it is concluded that WECs with larger diameter-to-draft ratios (DDRs) are

\section{REFERENCE}

Falcão, A. F. O. (2010). Wave energy utilization: a review of the technologies. Renew. Sustain. Energy Rev. 14 (3), 899-918. doi:10.1016/j.rser.2009.11.003

Conflict of Interest: The authors declare that the research was conducted in the absence of any commercial or financial relationships that could be construed as a potential conflict of interest. found to have a relatively smaller viscous effect and achieve effective energy conversion in a broader frequency range. More conclusions with different types of bottoms can be found in the article.

In the end, we would like to thank all the contributing authors and reviewers for their invaluable thoughts and insightful discussions. We also sincerely appreciate the journal editors and the publication team behind it. Without their hard effort, this article collection would never become possible.

\section{AUTHOR CONTRIBUTIONS}

YL drafted the article, followed by the improvements made by the four coauthors before submission. All authors listed have made a substantial, direct, and intellectual contribution to the work and approved it for publication.

\section{ACKNOWLEDGMENTS}

The first author specially thanks the financial support provided by Grant-in-Aid for Early-Career Scientists (JSPS KAKENHI grant number JP18K13939) during the course of the research topic.

Copyright (c) 2020 Liu, Ding, Zhou, Cong and Zheng. This is an open-access article distributed under the terms of the Creative Commons Attribution License (CC BY). The use, distribution or reproduction in other forums is permitted, provided the original author(s) and the copyright owner(s) are credited and that the original publication in this journal is cited, in accordance with accepted academic practice. No use, distribution or reproduction is permitted which does not comply with these terms. 